\title{
Retrospective imaging study on the diagnosis of pathological false positive iodine-131 scans in patients with thyroid cancer
}

\author{
QIANG JIA $^{1}$, ZHAOWEI MENG ${ }^{1}$, JIAN TAN ${ }^{1}$, GUIZHI ZHANG ${ }^{1}$, YAJING HE ${ }^{1}$, HAORAN SUN $^{2}$, \\ CHUNSHUI YU ${ }^{2}$, DONG LI ${ }^{2}$, WEI ZHENG ${ }^{1}$, RENFEI WANG $^{1}$, SHEN WANG $^{1}, \mathrm{XUE} \mathrm{LI}^{1}$, \\ JIANPING ZHANG ${ }^{1}$, TIANPENG HU ${ }^{1}$, NA LIU ${ }^{1}$ and ARUN UPADHYAYA ${ }^{1}$ \\ Departments of ${ }^{1}$ Nuclear Medicine and ${ }^{2}$ Radiology, Tianjin Medical University General Hospital, \\ Heping, Tianjin 300052, P.R. China
}

Received October 15, 2014; Accepted September 1, 2015

DOI: $10.3892 /$ etm.2015.2744

\begin{abstract}
Iodine-131 (I-131) therapy and post-therapy I-131 scanning are essential in the management of differentiated thyroid cancer (DTC). However, pathological false positive I-131 scans can lead to misdiagnosis and inappropriate I-131 treatment. This retrospective study aimed to investigate the best imaging modality for the diagnosis of pathological false positive I-131 scans in a DTC patient cohort, and to determine its incidence. DTC patient data archived from January 2008 to January 2010 was retrieved. Post-therapeutic I-131 scans were conducted and interpreted. The imaging modalities of magnetic resonance imaging (MRI), computed tomography and ultrasonography were applied and compared to check all suspected lesions. Biopsy or needle aspiration was conducted for patients who consented to the acquisition of histopathological confirmation. Data for 156 DTC patients were retrieved. Only 6 cases of pathological false-positives were found among these (incidence, $3.85 \%$ ), which included 3 cases of thymic hyperplasia in the mediastinum, 1 case of pleomorphic adenoma in the parapharyngeal space and 1 case of thyroglossal duct cyst in the neck. MRI was demonstrated as the best imaging modality for diagnosis due to its superior soft tissue resolution. However, no imaging modality was able to identify the abdominal false positive-lesions observed in 2 cases, one of whom also had thymic hyperplasia. In conclusion, pathological false positive I-131 scans occurred with an incidence of $3.85 \%$. MRI was the best imaging modality for diagnosing these pathological false-positives.
\end{abstract}

Correspondence to: Professor Zhaowei Meng, Department of Nuclear Medicine, Tianjin Medical University General Hospital, 154 Anshan Road, Heping, Tianjin 300052, P.R. China

E-mail: jamesmencius@163.com

Key words: differentiated thyroid cancer, iodine-131 scan, pathological false positive, magnetic resonance imaging

\section{Introduction}

Iodine-131 (I-131) therapy and post-therapy I-131 scanning have been accepted as essential components of the overall management of patients with differentiated thyroid cancer (DTC) for over six decades $(1,2)$. The finding of any positive lesion on an I-131 scan is often a decisive indicator for the administration of subsequent I-131 therapies. Generally, I-131 scanning has a sensitivity of $60-80 \%$ and specificity of $>90 \%$ (3-6). Despite this remarkable specificity, false positive uptakes on I-131 scans do occur, which can be misdiagnosed as residual or metastatic DTC lesions, and lead to inappropriate I-131 therapies. Since the risk of secondary malignancies in DTC survivors treated with I-131 is slightly increased compared with that of DTC survivors not treated with I-131 (7), and this risk is dose-related (8), it is important to identify false positive I-131 scans in order to avoid unnecessary exposure to radiation.

In the literature, the causes of false positive results are reported to include contamination, physiological uptake and unrelated pathological uptake (3,6,9-13). Regarding saliva, perspiration and urine contamination, with adequate experience, the clinical practices responsible for this may be identified. It may be necessary to wash certain body parts or change contaminated clothes. Physiological uptake in salivary glands, gastrointestinal and urinary tracts has a characteristic appearance, and can often be recognized unequivocally. The true challenge is the diagnosis of pathological false positive I-131 scans. Therefore, the main purpose of this retrospective investigation was to determine the best imaging modality for identifying the pathology in patients with pathological false positive I-131 scans by the investigation of a DTC patient cohort, and to determine the incidence of pathological false positives.

\section{Materials and methods}

Patients. This study was conducted as a collaboration between the Nuclear Medicine Department and Radiology Department of Tianjin Medical University General Hospital (Tianjin, China). The data of patients with DTC archived from January 2008 to January 2010 was retrieved. The study was approved by the Institutional Review Board and Ethics Committee of 
Tianjin Medical University. Written informed consent was obtained from all patients.

Treatment procedures. The 2006 guidelines (1) and then the 2009 revised guidelines (2) from the American Thyroid Association (ATA) were used to treat and monitor these patients. Briefly, thyroid remnant ablation was performed with I-131 activities of 80-100 $\mathrm{mCi}$. If residual thyroid persisted further ablations were conducted. Then, empiric I-131 therapy (100-200 mCi) was administered if cervical, pulmonary or skeletal metastases were found. I-131 therapy required thyrotropin stimulation for which the patients were prepared by thyroxine withdrawal for 2-3 weeks along with a low-iodine diet. Post-therapy I-131 whole body scans were typically conducted $\sim 1$ week after I-131 therapies, using a dual-detector single-photon emission computed tomography (SPECT) instrument equipped with high-energy parallel-hole collimators (Discovery VH; GE Healthcare, Milwaukee, WI, USA). Serum thyroglobulin (Tg) and thyroglobulin antibody $(\mathrm{TgAb})$ levels were measured by immunometric assays under thyrotropin-stimulated status during I-131 therapies (Immulite 2000; Siemens Medical Solutions Diagnostics, Los Angeles, CA, USA). Simulated levels of $\mathrm{Tg}<2.0 \mathrm{ng} / \mathrm{ml}$ were considered as an indication of DTC lesion-free status, and $\mathrm{Tg} \geq 2.0 \mathrm{ng} / \mathrm{ml}$ as an indication of DTC lesion persistence; this cutoff value is advocated by ATA guidelines $(1,2)$. A panel of eight nuclear medicine physicians took direct charge of the above procedures, and independently interpreted the post-therapy I-131 scans. Any persistent I-131-avid lesion after complete thyroid remnant ablation was considered as a positive lesion. The protocol for any susceptive positive finding was as follows. When contamination was suspected, another I-131 scan was applied after washing body parts or changing into new clothes to confirm the results. Physiological uptake in the salivary glands or in the gastrointestinal or urinary tract was determined by consensus of the panel. When pathological positive lesions were identified, they were marked and later compared with the radiological findings with more attention.

Imaging procedures. All magnetic resonance imaging (MRI) examinations were performed with a Signa HDx scanner using a 3-T superconducting magnet (GE Healthcare). The imaging sequences included T1-, T2-weighted fast spin echo either with or without fat suppression. All computed tomography (CT) scans were performed using a 64-slice spiral CT machine (LightSpeed VCT; GE Healthcare, Waukesha, WI, USA). Photographic images of scans were captured at windows appropriate for viewing the mediastinum and the lung. High-resolution gray-scale ultrasonography (US) was conducted with real-time equipment (Vivid Five; GE Vingmed Ultrasound, Horten, Norway), using a 10-MHz linear-array transducer. Positron emission tomography (PET) was not included as an imaging modality. In addition to its prohibitive expense, the application of PET is most suitable for the localization of lesions in patients with DTC who are Tg-positive and I-131 scan false negative $(1,2)$.

Analysis of false positive scans. The aim of the study was to identify false positive I-131 scans. A panel of three radiologists took charge of the radiological procedures, and independently interpreted the images. Consensus concerning the diagnosis of non-DTC related lesions was reached by majority. Then radiological images and diagnoses were supplied to the Nuclear Medicine Panel for further assessments. Biopsy or needle aspiration results were conducted for patients who consented to the acquisition of histopathological confirmation.

Finally, the characteristics of the DTC patients with pathological false positive I-131 scans were summarized and compared in an attempt to determine the best imaging modality for diagnosis.

\section{Results}

Patient data. From the database, 156 patients with DTC [142 papillary thyroid cancer (PTC) and 14 follicular thyroid cancer (FTC)] were retrieved. The mean age was $47.23 \pm 13.47$ years. Altogether, there were 494 I-131 therapies and the same number of subsequent post-therapy I-131 scans.

Pathological false positive cases. Among the patients, 6 cases were identified to have pathological false positive lesions. The incidence of pathological false positive I-131 scans in the DTC patient cohort was $3.85 \%$ (6/156 cases). All 6 cases had PTC pathology.

Three PTC cases (cases 1-3) had stimulated Tg levels $<2.0 \mathrm{ng} / \mathrm{ml}$, yet exhibited I-131-avid lesions in the anterior mediastinum on I-131 scans, which were considered as false positive lesions (Figs. 1-3). Case 1 also demonstrated a false positive I-131-avid lesion in the right lower abdomen (Fig. 1). Case 4, with a Tg level $<2.0 \mathrm{ng} / \mathrm{ml}$, also had a false positive lesion in the right lower abdomen. Case 5 had a stimulated $\mathrm{Tg}$ level of $10.7 \mathrm{ng} / \mathrm{ml}$ following complete thyroid remnant ablation during the second I-131 therapy, when cervical lesions and a large oropharyngeal lesion were demonstrated on the I-131 scan. Following three additional I-131 therapies the cervical lesions almost disappeared yet the oropharyngeal lesion persisted without change in shape and uptake ability, and was considered as false positive (Fig. 4). Case 6 had a stimulated Tg level between 3.5 and $4.1 \mathrm{ng} / \mathrm{ml}$, yet a disproportionately intense I-131-avid cervical lesion was displayed without any change in two consecutive I-131 therapies after thyroid remnant ablation (Fig. 5).

Evaluation of imaging modalities. Characteristics of the false positive I-131 scan cases are listed in Table I. MRI was demonstrated as the best imaging modality for diagnosis due to superior soft tissue resolution. US was shown to be the least useful, because thymus hyperplasia and parapharyngeal pleomorphic adenoma were not accessible. No imaging modality was able to locate false positive sites in the abdomen. MRI, CT or US did not detect lesions in other DTC patients as non-DTC lesions by the Radiology Panel, and no other pathological false positive I-131 scans were identified by the Nuclear Medicine Panel.

\section{Discussion}

The trapping of I-131 by DTC lesions allows the acquisition of scans to identify local and distant metastases. The challenge to the clinicians is to tailor the treatment to be vigorous enough to eradicate DTC lesions but not to cause unnecessary morbidity. The correct interpretation of I-131 scans is critical in the proper management of patients with DTC, which requires a thorough 
A

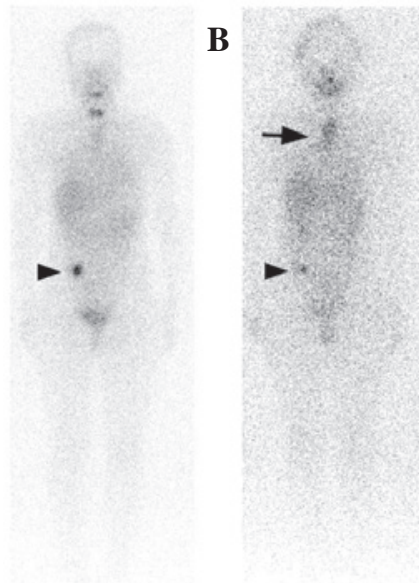

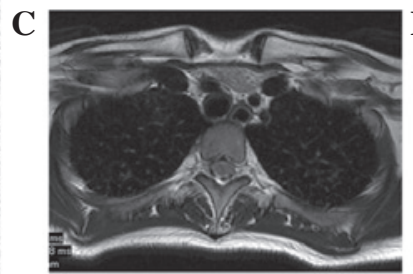
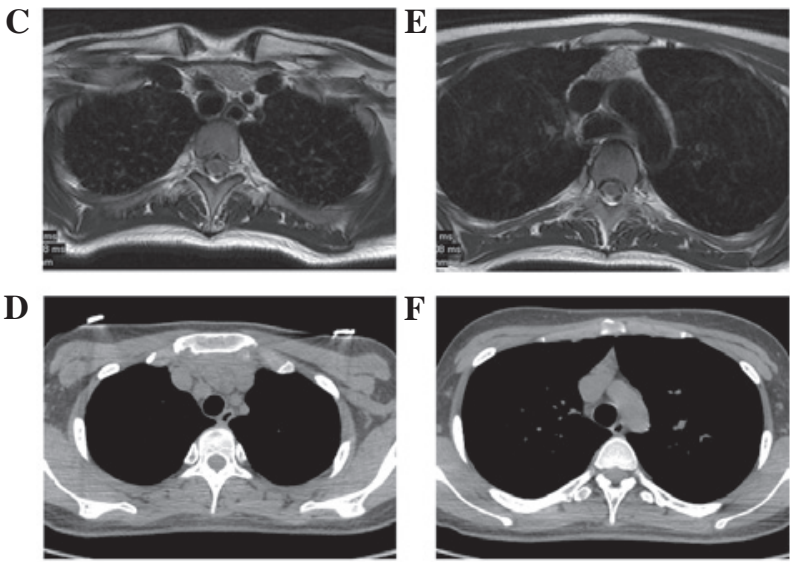

Figure 1. Case 1. A 22-year-old female PTC patient underwent three I-131 therapies. A I-131 false positive lesion was found in the right lower abdomen on the (A) second and (B) third post-therapy I-131 scans (arrowheads), however no imaging modality was able to detect and diagnose the lesion. (B) A large false positive lesion in the mediastinum was identified on the I-131 scan after the third therapy. The patient had a Tg level of $1.1 \mathrm{ng} / \mathrm{ml}$ under TSH stimulation. (C and D) On T2-weighted MRI images the signal intensity of the lesion was intermediate, which was lower than that of the adjacent fat tissue. (E and F) On CT images, the density of the lesion was higher than that of the adjacent fat tissue. MRI displayed the lesion with better contrast than that of CT, based on which bilaterally lobed thymic hyperplasia was diagnosed. Mediastinoscopy with biopsy confirmed the diagnosis. MRI and CT images were taken during the third I-131 therapy. PTC, papillary thyroid cancer; I-131, iodine-131; Tg, thyroglobulin; TSH, thyroid-stimulating hormone; MRI, magnetic resonance imaging; CT, computed tomography.

A

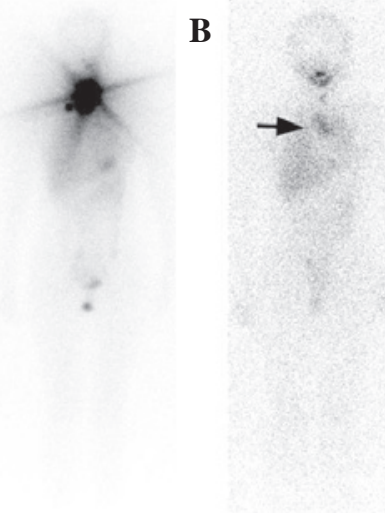

C
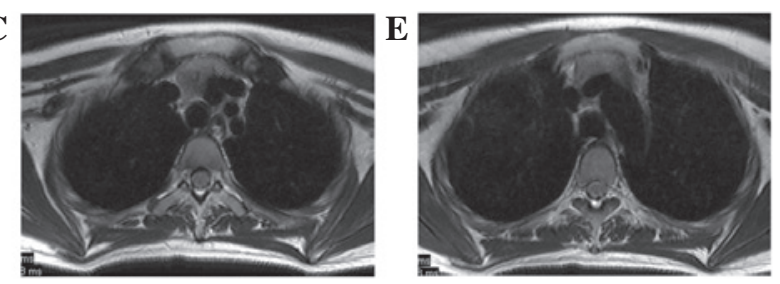

D

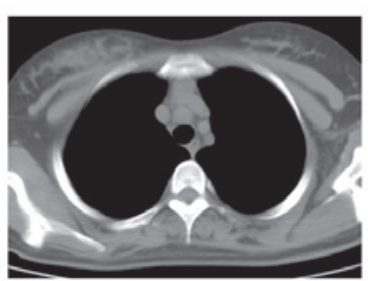

$\mathbf{F}$

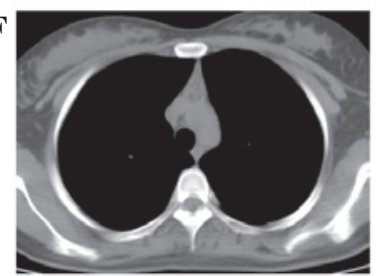

Figure 2. Case 2. A 19-year-old female PTC patient received two I-131 therapies. (A) The first post-therapy I-131 scan demonstrated a large thyroid remnant. (B) The second post-therapy I-131 scan indicated successful ablation of the thyroid remnant with a Tg level $<0.9 \mathrm{ng} / \mathrm{ml}$ under TSH stimulation, yet a false positive mediastinum lesion was visible (arrow). (C and D) On T2-weighted MRI images, a pyramidally shaped lesion with intermediate signal intensity (lower than that of adjacent fat tissue) was revealed. (E and F) CT images also exhibited the pyramidally shaped lesion with higher density than fat tissue. Since CT acquisition was finished during one suspended inspiration, the thoracic cavity was more extended than that in MRI images and the pyramidal shape of the lesion seemed more evident. MRI displayed better resolution of the lesion than CT, and consequently thymic hyperplasia was diagnosed. Mediastinoscopy biopsy also confirmed the diagnosis. MRI and CT images were taken during the second I-131 therapy. PTC, papillary thyroid cancer; I-131, iodine-131; Tg, thyroglobulin; TSH, thyroid-stimulating hormone; MRI, magnetic resonance imaging; CT, computed tomography.

knowledge and understanding of all potential confounding phenomena. False positive findings on I-131 scans can be classified into contamination, physiological uptake and pathological uptake $(3,4,6,9-13)$. Pathological false positive uptake of I-131, ranging from non-thyroidal neoplasm to inflammation or infection, epitomizes the diagnostic difficulties that clinicians could confront in this perspective when managing patients with DTC. Ideally, the identification of false-positive results should be made through conservative means whenever possible $(3,4,6,9-13)$.

For patients with DTC, the finding of a mediastinal mass represents a diagnostic dilemma, and the distinction between thymic hyperplasia and DTC metastasis must be made correctly. Notably, Niendorf et al (14) once reported a high prevalence of $33 \%$ for thymic hyperplasia in patients with DTC on the basis of CT diagnostic criteria, which was more commonly found in the subgroup of patients with DTC who did not have metastasis. However, a study by Davidson and Davidson (15) demonstrated that 6/175 DTC patients exhibited I-131 uptake by the thymus, and they were found to be disease-free during the follow-up. These facts indicated that only some DTC patients with thymic hyperplasia could actually result in a false-positive conundrum in I-131 imaging. The mechanism in this respect may be explained by 
A

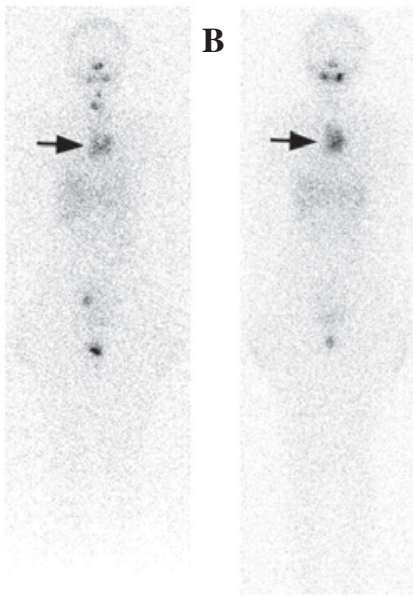

$\mathbf{C}$

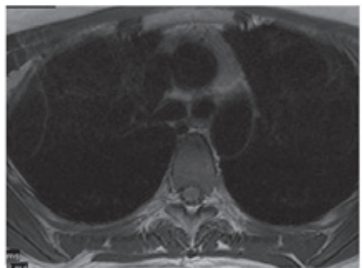

D

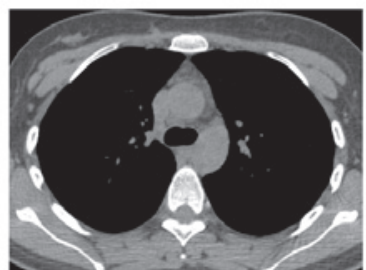

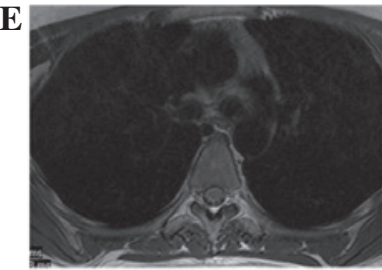

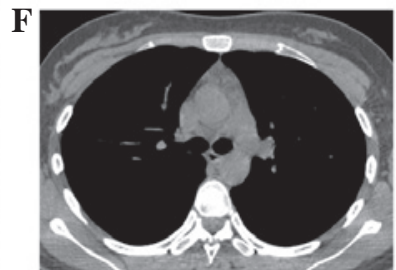

Figure 3. Case 3. A 23-year-old female PTC patient received three I-131 therapies. (A) The second post-therapy I-131 scan demonstrated a small amount of thyroid remnant, yet a large mediastinum lesion was demonstrated (arrow), which was much clearer on (B) the third post-therapy I-131 scan when thyroid remnant was completely ablated (arrow). The TSH-stimulated Tg levels were 1.2 and $1.0 \mathrm{ng} / \mathrm{ml}$ during these I-131 scans. (C and D) On T2-weighted MRI images, a soft tissue lesion with intermediate signal intensity tilted to the left side of the anterior superior mediastinum was shown. (E and F) Although CT images also showed the lesion, it was obscure compared with MRI and easily overlooked without careful reading. Thymic hyperplasia was diagnosed primarily on MRI images. Consent for biopsy was not given. MRI and CT images were taken during the third I-131 therapy. PTC, papillary thyroid cancer; I-131, iodine-131; Tg, thyroglobulin; TSH, thyroid-stimulating hormone; MRI, magnetic resonance imaging; CT, computed tomography.

A

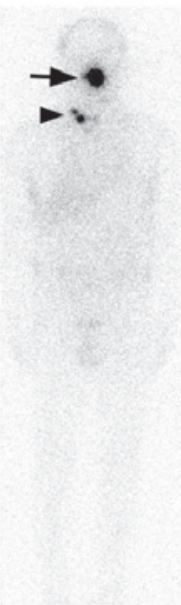

B

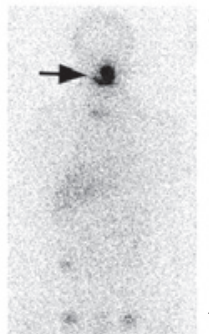

$\mathbf{C}$
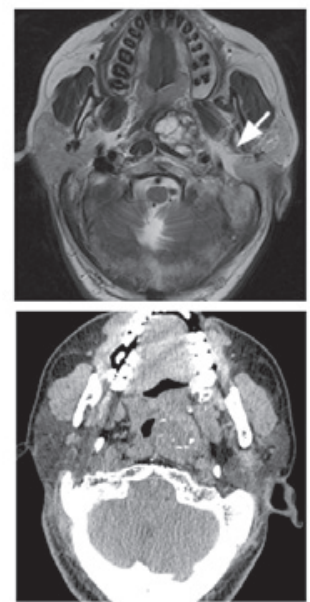

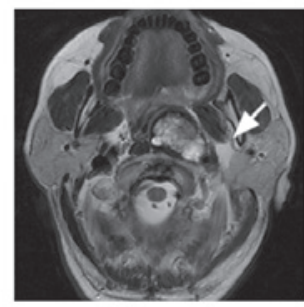

F

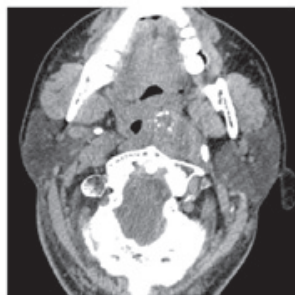

Figure 4. Case 5. A 43-year-old male PTC patient was given five I-131 therapies. (A) Cervical lymph node metastases (arrowhead) and an oropharynx lesion (arrow) were revealed following complete thyroid remnant ablation on the second post-therapy I-131 scan while the TSH-stimulated Tg level was $10.7 \mathrm{ng} / \mathrm{ml}$. (B) Another $3 \mathrm{I}-131$ treatment virtually destroyed all cervical lymph node metastases reducing the Tg level to $2.8 \mathrm{ng} / \mathrm{ml}$ under stimulation, yet the oropharynx lesion remained the same in shape and I-131 uptake ability. (C and D) T2-weighted MRI demonstrated a large oval-shaped lesion with heterogeneous signal intensity almost obstructing the oropharynx. This lesion was in the pre-styloid compartment of the parapharyngeal space, and a clear fatty plane was shown between the lesion and the parotid deep lobe (white arrows), indicating it was separated from the parotid gland. (E and F) CT images displayed focal calcifications in the mass and the position of styloid process, suggesting its location in the pre-styloid space. Generally, MRI provided more information than CT about pleomorphic content, location, margins of the lesion and its relationships with surrounding structures; based on which pleomorphic adenoma was diagnosed. Biopsy histopathology confirmed this diagnosis. MRI and CT images were taken during the fifth I-131 therapy. PTC, papillary thyroid cancer; I-131, iodine-131; Tg, thyroglobulin; TSH, thyroid-stimulating hormone; MRI, magnetic resonance imaging; CT, computed tomography.

the presence of the sodium iodine symporter (16); however, compared with the thyroid gland, the capacity to transport and concentrate iodine has been found to be diminished in extrathyroidal tissues, and it appears that the absence of remnant thyroid or metastasis plus higher I-131 activities could increase the probability of thymus visualization on post-therapeutic I-131 scans $(17,18)$. In the present study, 3/156 patients demonstrated a positive uptake of I-131 by the thymus while their $\mathrm{Tg}$ results were negative. All of the patients were young and DTC lesion-free. Both MRI and CT displayed soft tissue prominences that affected the bilateral lobes (Fig. 1), were pyramidally shaped (Fig. 2) or deviated to the left of the anterior mediastinum (Fig. 3), indicating thymic hyperplasia. However, MRI was superior to CT because of its clearer soft tissue resolution (e.g., thymic tissue vs. fat tissue) and distinctive large blood vessel landmarks due to the flow void phenomenon. However, there is a limitation for MRI, in that the acquisition time is much longer than that of 64-slice CT, so misregistration artifacts from respiration might obscure the lesion on MRI images. Since 64-slice 
A

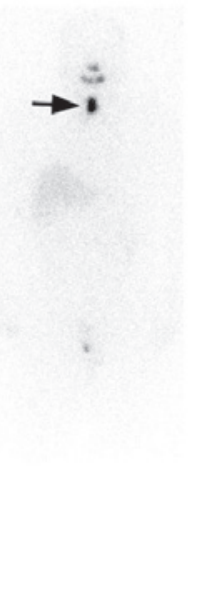

B

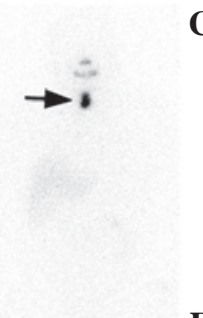

C

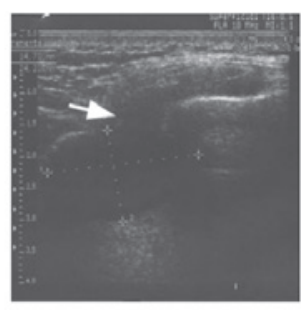

D

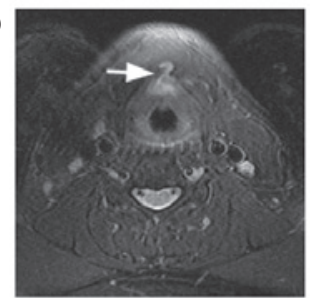

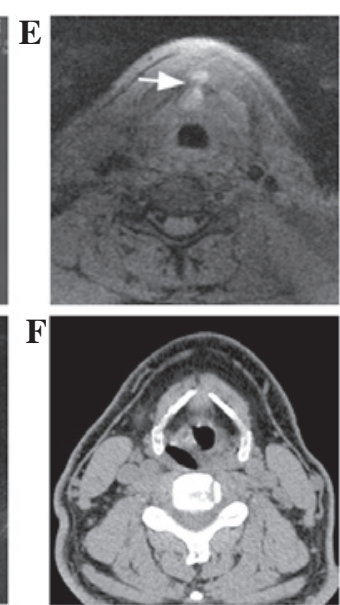

Figure 5. Case 6. A 44 year-old male PTC patient was treated with a total of three I-131 therapies. Consequent I-131 scans after the (A) second and (B) third I-131 treatments showed successful thyroid remnant ablation, yet an oval lesion in the midline of the neck was exhibited with its location, shape and intensity unchanged during the follow-up (arrows). TSH-stimulated Tg levels were 4.1, 3.5 and $3.8 \mathrm{ng} / \mathrm{ml}$ for the three therapies. (C) US demonstrated a dumbbell-shaped cyst lesion with the upper part posterior to the hyoid bone and the lower part anterior to the thyroid cartilage and isthmus through thyrohyoid membrane (white arrow). Due to interference by hyoid bone and thyroid cartilage, the lesion margins were not so sharp and clear on US images. (D and E) T1- and T2-weighted MRI images with fat suppression exhibited the uniquely shaped lesion much more clearly, particularly the penetration of the lesion isthmus through thyrohyoid membrane (white arrows). The lesion demonstrated slightly high signal intensity and high signal intensity on fat-suppressed T1- and T2-weighted images, respectively, with homogeneous distribution, reflecting the protein-rich fluid content of the cyst. Thyroglossal duct cyst was diagnosed by MRI. (F) However, it was difficult to diagnose by CT alone, because the density of the lesion was almost identical to that of the adjacent soft tissue structures. Aspiration pathology confirmed MRI findings concerning the content of the lesion.MRI, CT and US images were taken during the third I-131 therapy. PTC, papillary thyroid cancer; I-131, iodine-131; Tg, thyroglobulin; TSH, thyroid-stimulating hormone; US, ultrasound; MRI, magnetic resonance imaging; $\mathrm{CT}$, computed tomography.

CT data is acquired during one suspended inspiration, the thoracic cavity is more extended in CT images than on MRI images. As standard protocols for CT scanning of the thorax, patients are requested to cross their arms over the head, yet MRI acquisition requires patients to put their arms beside their body due to the narrowness of the gantry; this can often result in alterations in the relative positioning of the breasts and clavicles on CT images.

Tumors in the parapharyngeal space are very rare and account for only $0.5 \%$ of head and neck neoplasms; these usually present a formidable challenge for preoperative diagnosis as well as appropriate surgical treatment (19-22). This space is divided into pre-styloid and post-styloid compartments by a styloid diaphragm extending from the styloid process to the tensor-vascular-styloid fascia. In pre-styloid space, pleomorphic adenoma is the most common benign neoplasm. Previous studies have demonstrated that MRI is better than CT in detecting anatomical complexity, tissue diversity, relation to the internal carotid artery and origin of pleomorphic adenoma, and is the choice of examination (19-22). Furthermore, if MRI shows a fatty plane between pleomorphic adenoma and the parotid deep lobe, as shown in the present case 5 (Fig. 4), it indicates that the tumor is separated from the parotid deep lobe and originates from a minor salivary gland. Although CT is slightly inferior to MRI in soft tissue evaluation, it can better depict the styloid process and focal calcified degeneration (Fig. 4), which is also often observed in pleomorphic adenoma (23). When imaging studies demonstrate a parapharyngeal lesion with no vascular nature, biopsy or fine needle aspiration can provide very accurate histopathological diagnosis (19,21-22). In the present investigation, case 5 was firmly diagnosed as pleomorphic adenoma by MRI in the pre-styloid space, and CT provided additional information of focal calcification. Following complete ablation of the thyroid remnant, cervical lymph node metastases and a parapharyngeal pleomorphic adenoma were revealed. When I-131 therapies destroyed almost all cervical metastases, the pleomorphic adenoma persisted without change in shape and uptake intensity, causing evident false positive I-131 uptake. To the best of our knowledge, there are no previous reports of such a pathological false-positive. Our hypothesis of the underlying mechanism is, as a benign tumor originating from the salivary gland, pleomorphic adenoma retains I-131 uptake ability, which is mediated by the expression of sodium iodine symporter (16).

The concurrence of ectopic thyroid, thyroglossal duct remnant or thyroglossal duct cyst with DTC has previously been reported (24-26). Any dormant ectopic thyroid tissue that is left along the thyroglossal duct during the embryological development and migration of the thyroid primordium to form the thyroid gland can be augmented by TSH stimulation under the hypothyroidism status during I-131 therapy. They can be mistaken for DTC metastases and the false positive I-131 scans could be significant diagnostic pitfalls (24-26). Anatomical imaging modalities are very important for differential diagnoses, which were compared in case 6 in the present study. Due to high soft tissue resolution, MRI was able to clearly display the dumbbell-shaped thyroglossal duct cyst with isthmus penetrating through the thyrohyoid membrane, particularly in the fat-suppressed T2-weighted sequence (Fig. 5). Fluid content rich in protein was diagnosed in the cyst by MRI, which was confirmed by needle aspiration pathology. US had the great advantage of detecting cervical lesions, and 


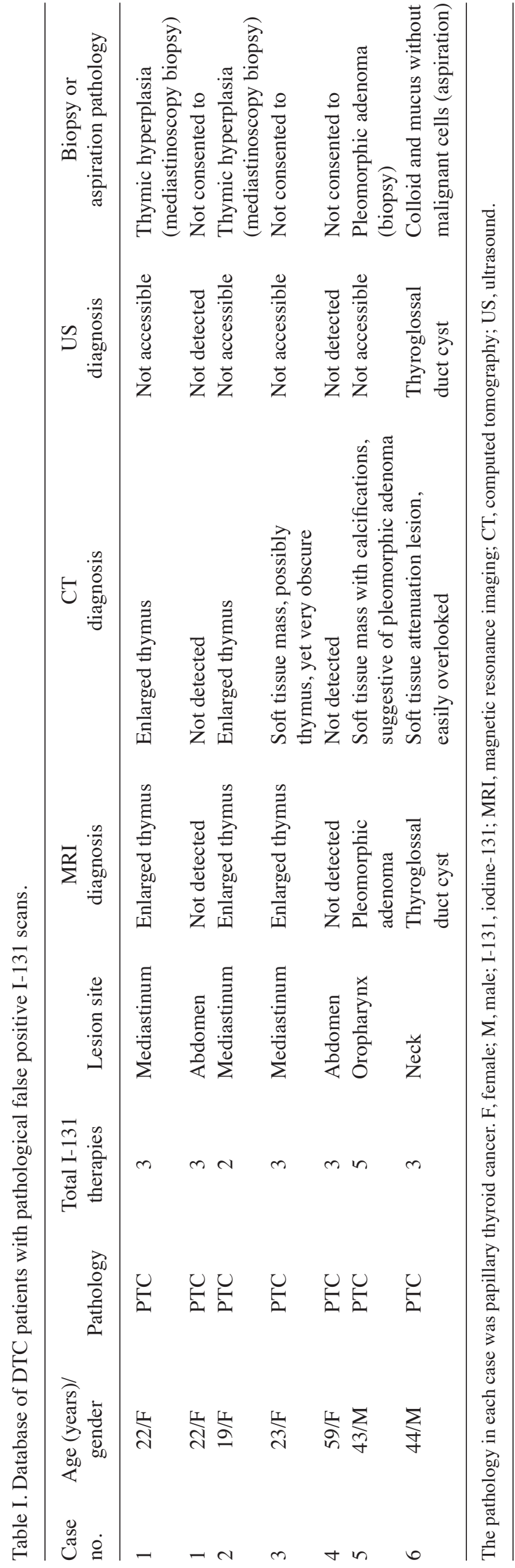

also revealed the fluid-content filled cyst in case 6. Although the focal margins of the lesion were not very sharp owing to interference by hyoid bone and thyroid cartilage, the diagnosis by US was quite conclusive. However, it would be relatively difficult to diagnose the thyroglossal duct cyst by CT alone in case 6 , particularly if CT images were not carefully read or other imaging modality assistance was not consulted, since the density of the thyroglossal duct cyst was almost the same as that of the adjacent soft tissue structures (Fig. 5). However, if the diagnosis was established, CT would exhibit the advantage of presenting the complex relationship of the cyst with hyoid bone and thyroid cartilage.

US is, by far, the most frequently used imaging procedure in monitoring residual or recurrent thyroid cancer in the central and lateral neck. However, the diagnostic capabilities of US in the parapharyngeal, retropharyngeal and mediastinal areas are limited by interference from bone and air. MRI and CT are of particular interest since they provide excellent anatomical images for these areas. Compared with CT, MRI causes negligible exposure to radiation and provides superior soft tissue resolution, and MRI avoids the limitation inherent to iodinated contrast agents of CT in the imaging of patients with DTC who are undertaking I-131 therapies. In fact, MRI has been demonstrated to be a very efficient modality for the detection of persistent or recurrent DTC in cervical, mediastinal, parapharyngeal and retropharyngeal spaces (27-29). In the current investigation, MRI exhibited superior capability compared with that of CT and US in the diagnosis of pathological false positive I-131-avid lesions, namely, thymic hyperplasia in the anterior mediastinum, pleomorphic adenoma in the parapharyngeal space and thyroglossal duct cyst in the neck. In addition, all imaging modalities were of limited usage in the identification of abdominal false positive I-131 lesions in this cohort (case 1 and 4), probably because the lesions were too small, although regional inflammation of the right lower abdomen, such as chronic appendicitis, colitis or oophoritis was suspected.

A limitation of the present study is the comparatively small size of the cohort, which intrinsically could only encompass a restricted number of pathological I-131 false positive cases. Although the retrospective analysis is very laborious, a further study over a much longer period is planned.

In conclusion, pathological false positive I-131 scans occurred with an incidence of $3.85 \%$ in the current DTC patient cohort, which included thymic hyperplasia in the mediastinum (3 cases), pleomorphic adenoma in the parapharyngeal space (1 case) and thyroglossal duct cyst in the neck (1 case). MRI was demonstrated to be the best imaging modality for diagnosing these pathological false-positives.

\section{Acknowledgements}

This investigation was supported by National Key Clinical Specialty Project (awarded to the Departments of Nuclear Medicine and Radiology). This study was also supported by Tianjin Medical University New Century Excellent Talent Program; Young and Middle-aged Innovative Talent Training Program from Tianjin Education Committee; and Talent Fostering Program (the 131 Project) from Tianjin Human Resources and Social Security Bureau (all awarded to Zhaowei Meng). 


\section{References}

1. Cooper DS, Doherty GM, Haugen BR, Kloos RT, Lee SL, Mandel SJ, Mazzaferri EL, McIver B, Sherman SI and Tuttle RM; American Thyroid Association Guidelines Taskforce: Management guidelines for patients with thyroid nodules and differentiated thyroid cancer. Thyroid 16: 109-142, 2006

2. American Thyroid Association (ATA) Guidelines Taskforce on Thyroid Nodules and Differentiated Thyroid Cancer; Cooper DS, Doherty GM, Haugen BR, Kloos RT, Lee SL, Mandel SJ, Mazzaferri EL, McIver B, Pacini F, Schlumberger M, et al: Revised American Thyroid Association management guidelines for patients with thyroid nodules and differentiated thyroid cancer. Thyroid 19: 1167-1214, 2009.

3. Carlisle MR, Lu C and McDougall IR: The interpretation of ${ }^{131} \mathrm{I}$ scans in the evaluation of thyroid cancer, with an emphasis on false positive findings. Nucl Med Commun 24: 715-735, 2003.

4. van Sorge-van, Boxtel RA, van Eck-Smit BL and Goslings BM: Comparison of serum thyroglobulin, ${ }^{131} \mathrm{I}$ and ${ }^{201} \mathrm{Tl}$ scintigraphy in the postoperative follow-up of differentiated thyroid cancer Nucl Med Commun 14: 365-372, 1993.

5. Lubin E, Mechlis-Frish S, Zatz S, Shimoni A, Segal K, Avraham A, Levy R and Feinmesser R: Serum thyroglobulin and iodine-131 whole-body scan in the diagnosis and assessment of treatment for metastatic differentiated thyroid carcinoma. J Nucl Med 35: 257-262, 1994

6. McDougall IR: Whole-body scintigraphy with radioiodine-131. A comprehensive list of false-positives with some examples. Clin Nucl Med 20: 869-875, 1995.

7. Sawka AM, Thabane L, Parlea L, Ibrahim-Zada I, Tsang RW, Brierley JD, Straus S, Ezzat S and Goldstein DP: Second primary malignancy risk after radioactive iodine treatment for thyroid cancer: A systematic review and meta-analysis. Thyroid 19: 451-457, 2009.

8. Rubino C, de Vathaire F, Dottorini ME, Hall P, Schvartz C, Couette JE, Dondon MG, Abbas MT, Langlois C and Schlumberger M: Second primary malignancies in thyroid cancer patients. Br J Cancer 89: 1638-1644, 2003.

9. Shapiro B, Rufini V, Jarwan A, Geatti O, Kearfott KJ, Fig LM Kirkwood ID and Gross MD: Artifacts, anatomical and physiological variants, and unrelated diseases that might cause false-positive whole-body 131-I scans in patients with thyroid cancer. Semin Nucl Med 30: 115-132, 2000.

10. Sutter CW, Masilungan BG and Stadalnik RC: False-positive results of I-131 whole-body scans in patients with thyroid cancer. Semin Nucl Med 25: 279-282, 1995.

11. Greenler DP and Klein HA: The scope of false-positive iodine-131 images for thyroid carcinoma. Clin Nucl Med 14: 111-117, 1989.

12. Bakheet SM and Hammami MM: False-positive radioiodine whole-body scan in thyroid cancer patients due to unrelated pathology. Clin Nucl Med 19: 325-329, 1994.

13. Mitchell G, Pratt BE, Vini L, McCready VR and Harmer CL: False positive 131I whole body scans in thyroid cancer. Br J Radiol 73: 627-635, 2000.

14. Niendorf ER, Parker JA, Yechoor V, Garber JR and Boiselle PM: Thymic hyperplasia in thyroid cancer patients. J Thorac Imaging 20: 1-4, 2005.
15. Davidson J and McDougall IR: How frequently is the thymus seen on whole-body iodine-131 diagnostic and post-treatment scans? Eur J Nucl Med 27: 425-430, 2000.

16. Spitzweg C, Joba W, Eisenmenger W and Heufelder AE: Analysis of human sodium iodide symporter gene expression in extrathyroidal tissues and cloning of its complementary deoxyribonucleic acids from salivary gland, mammary gland and gastric mucosa. J Clin Endocrinol Metab 83: 1746-1751, 1998.

17. Meller $\mathbf{J}$ and Becker $\mathrm{W}$ : The human sodium-iodine symporter (NIS) as a key for specific thymic iodine-131 uptake. Eur J Nucl Med 27: 473-474, 2000

18. Michigishi T, Mizukami Y, Shuke N, Yokoyama K, Noguchi M, Watanabe Y, Matsui O, Aburano T, Tonami N and Hisada K: Visualization of the thymus with therapeutic doses of radioiodine in patients with thyroid cancer. Eur J Nucl Med 20: 75-79, 1993.

19. Bozza F, Vigili MG, Ruscito P, Marzetti A and Marzetti F: Surgical management of parapharyngeal space tumours: Results of 10-year follow-up. Acta Otorhinolaryngol Ital 29: 10-15, 2009.

20. Dimitrijevic MV, Jesic SD, Mikic AA, Arsovic NA and Tomanovic NR: Parapharyngeal space tumors: 61 case reviews. Int J Oral Maxillofac Surg 39: 983-989, 2010.

21. Sergi B, Limongelli A, Scarano E, Fetoni AR and Paludetti G: Giant deep lobe parotid gland pleomorphic adenoma involving the parapharyngeal space. Report of three cases and review of the diagnostic and therapeutic approaches. Acta Otorhinolaryngol Ital 28: 261-265, 2008.

22. Papadogeorgakis N, Petsinis V, Goutzanis L, Kostakis G and Alexandridis C: Parapharyngeal space tumors: Surgical approaches in a series of 13 cases. Int J Oral Maxillofac Surg 39: 243-250, 2010

23. Shi H, Wang P, Wang S and Yu Q: Pleomorphic adenoma with extensive ossified and calcified degeneration: Unusual CT findings in one case. AJNR Am J Neuroradiol 29: 737-738, 2008.

24. Basaria S, Westra WH and Cooper DS: Ectopic lingual thyroid masquerading as thyroid cancer metastases. J Clin Endocrinol Metab 86: 392-395, 2001.

25. Zanotti-Fregonara P, Hindié E, Keller I, Calzada-Nocaudie M and Devaux JY: Scintigraphic visualization of glossal thyroid tissue during the follow-up of thyroid cancer patients. Clin Nucl Med 32: 911-914, 2007.

26. Li D, Meng Z, Zhang G, Yu T, Tan J and Dong F: Visualization of thyroglossal duct cyst in differentiated thyroid cancer patient. Clin Nucl Med 35: 499-504, 2010.

27. Kaplan SL, Mandel SJ, Muller R, Baloch ZW, Thaler ER and Loevner LA: The role of MR imaging in detecting nodal disease in thyroidectomy patients with rising thyroglobulin levels. AJNR Am J Neuroradiol 30: 608-612, 2009.

28. Gross ND, Weissman JL, Talbot JM, Andersen PE, Wax MK and Cohen JI: MRI detection of cervical metastasis from differentiated thyroid carcinoma. Laryngoscope 111: 1905-1909, 2001.

29. Toubert ME, Cyna-Gorse F, Zagdanski AM, Noel-Wekstein S, Cattan P, Billotey C, Sarfati E and Rain JD: Cervicomediastinal magnetic resonance imaging in persistent or recurrent papillary thyroid carcinoma: Clinical use and limits. Thyroid 9: e-597, 1999. 